\title{
Cooperation of sperm in two dimensions: Synchronization, attraction, and aggregation through hydrodynamic interactions
}

\author{
Yingzi Yang, Jens Elgeti, and Gerhard Gompper* \\ Theoretical Soft Matter and Biophysics Group, Institut für Festkörperforschung, Forschungszentrum Jülich, D52425 Jülich, Germany
}

(Received 1 August 2008; revised manuscript received 6 October 2008; published 3 December 2008)

\begin{abstract}
Sperm swimming at low Reynolds number have strong hydrodynamic interactions when their concentration is high in vivo or near substrates in vitro. The beating tails not only propel the sperm through a fluid, but also create flow fields through which sperm interact with each other. We study the hydrodynamic interaction and cooperation of sperm embedded in a two-dimensional fluid by using a particle-based mesoscopic simulation method, multiparticle collision dynamics. We analyze the sperm behavior by investigating the relationship between the beating-phase difference and the relative sperm position, as well as the energy consumption. Two effects of hydrodynamic interaction are found, synchronization and attraction. With these hydrodynamic effects, a multisperm system shows swarm behavior with a power-law dependence of the average cluster size on the width of the distribution of beating frequencies.
\end{abstract}

DOI: 10.1103/PhysRevE.78.061903

PACS number(s): 87.16.Qp, 82.70.-y, 87.17.Aa

\section{INTRODUCTION}

Sperm motility is important for the reproduction of animals. A healthy mature sperm of a higher animal species usually has a flagellar tail, which beats in a roughly sinusoidal pattern and generates forces that drive fluid motion. At the same time, the dynamic shape of the elastic flagellum is influenced by the fluid dynamics. The snakelike motion of the tail propels the sperm through a fluid medium very efficiently. In the past decades, the effort to quantitatively describe the fluid dynamics of sperm has been very successful $[1,2]$.

However, despite considerable progress in modeling sperm elementary structures and the behavior of a single sperm in a fluid medium $[3,4]$, relatively few studies have examined the fluid-dynamics coupling of sperm and other mesoscopic or macroscopic objects, e.g., the synchrony of beating tails $[5,6]$, the tendency of accumulation near substrates [7-10], etc. In nature, the local density of sperm is sometimes extremely high. For example, in mammalian reproduction, the average number of sperm per ejaculate is tens to hundreds of millions, so that the average distance between sperm is on the scale of ten micrometers-comparable to the length of their flagellum. The sperm are so close that the interaction between them is not negligible. In recent years, experiments [11-16] have revealed an interesting swarm behavior of sperm at high concentration, e.g., the distinctive aggregations or "trains" of hundreds of wood-mouse sperm $[14,15]$, or the vortex arrays of swimming sea urchin sperm on a substrate [16]. The mechanisms behind the abundant experimental phenomena are still unclear. In this paper, we focus on the hydrodynamic interaction between sperm and explain its importance for the cooperative behavior.

The higher animal sperm typically have tails with a length of several tens of micrometers. At this length scale, viscous forces dominate over inertial forces. Thus, the swimming motion of a sperm corresponds to the regime of low Rey-

\footnotetext{
*g.gompper@fz-juelich.de
}

nolds number [17]. Experimental observations of two paramecium cells swimming at low Reynolds number have shown that the changes in direction of motion between two cells are induced mainly by hydrodynamic forces [18]. Studies of model micromachines indicate that hydrodynamic interaction is significant when the separation distance is comparable to their typical size [19]. The hydrodynamic interaction between two rotating helices, such as bacterial flagella, has been investigated both experimentally [20] and theoretically $[21,22]$. An artificial microswimmer, which mimics the motion of a beating sperm, has been constructed from a red blood cell as head and a flagellumlike tail composed of chemically linked paramagnetic beads; the propulsion is then induced by a magnetically driven undulation of the tail [23]. Simulations have been employed to study the motion of a single of these artificial microswimmers [24], as well as the hydrodynamic interactions between two swimmers [25]. Even studies of a minimal swimming model of three linearly connected spheres [26] have shown a complicated cooperative behavior [27]. Thus, although there has been much progress on modeling and observing a single swimmer, the understanding on the hydrodynamic coupling behavior of dense system of swimmers is still poor.

In this paper, we focus on the cooperation behavior of sperm in two dimensions. Although real swimming spermatozoa are certainly three dimensional, the qualitatively similar phenomena, and the great saving of simulation time, makes it worthwhile to discuss the problem of cooperation in a viscous fluid in two dimensions. Furthermore, sperm are attracted to substrates in invitro experiments [7-10] and are therefore often swimming under quasi-two-dimensional condition (it has to be emphasized that hydrodynamic interactions in two dimensions and in three dimensions near a substrate are, of course, different). Thus, we construct a coarsegrained sperm model in two dimensions and describe the motion of the surrounding fluid by using a particle-based mesoscopic simulation method called multiparticle collision dynamics (MPC) [28,29]. This simulation method has been shown to capture the hydrodynamics and flow behavior of complex fluids over a wide range of Reynolds numbers very 
well [30,31], and is thus very suitable for the simulation of swimming sperm.

This paper is organized as follows. Section 2 gives a brief description of our sperm model and of the particle-based hydrodynamics approach. In order to understand a complex many-body system of microswimmers, a first-but-important step is to investigate the interaction between two swimming sperm. Thus, in Sec. III, we look at the cooperative behavior of two sperm. Two remarkable hydrodynamic effects, synchronization and attraction, are found and discussed in detail. In Sec. IV, we analyze the clustering behavior of multisperm systems. In particular, we consider a sperm system with a distribution of beating frequencies, and determine the dependence of the cluster size on the variance of the frequency distribution.

\section{SPERM MODEL AND MESOSCALE HYDRODYNAMICS}

\section{A. Multiparticle collision dynamics (MPC)}

MPC is a particle-based mesoscopic simulation technique to describe the complex fluid behaviors for a wide range of Reynolds numbers [30,31]. The fluid is modeled by $N$ point particles, which are characterized by their mass $m_{i}$, continuous space position $\mathbf{r}_{i}$, and continuous velocity $\mathbf{u}_{i}$, where $i$ $=1, \ldots, N$. In MPC simulations, time $t$ is discrete. During every time step $\Delta t$, there are two simulation steps, streaming and collision. In the streaming step, the particles do not interact with each other, and move ballistically according to their velocities

$$
\mathbf{r}_{i}(t+\Delta t)=\mathbf{r}_{i}(t)+\mathbf{u}_{i} \Delta t
$$

In the collision step, the particles are sorted into collision boxes of side length $a$ according to their position, and interact with all other particles in same box through a multibody collision. The collision step is defined by a rotation of all particle velocities in a box in a comoving frame with its center-of-mass. Thus, the velocity of the $i$ th particle in the $j$ th box after collision is

$$
\mathbf{u}_{i}(t+\Delta t)=\mathbf{u}_{\mathrm{c} . \mathrm{m} ., j}(t)+\mathfrak{R}_{j}(\alpha)\left[\mathbf{u}_{i}-\mathbf{u}_{\mathrm{c} . \mathrm{m} ., j}\right],
$$

where

$$
\mathbf{u}_{\mathrm{c} . \mathrm{m} ., j}(t)=\frac{\sum_{j} m_{i} \mathbf{u}_{i}}{\sum_{j} m_{i}}
$$

is the center-of-mass velocity of the $j$ th box and $\mathfrak{R}_{j}(\alpha)$ is a rotation matrix which rotates a vector by an angle $\pm \alpha$, with the sign chosen randomly. This implies that during the collision each particle changes the magnitude and direction of its velocity, but the total momentum and kinetic energy are conserved within every collision box. In order to ensure Galilean invariance, a random shift of the collision grid has to be performed [32,33].

The total kinematic viscosity $\nu$ is the sum of two contributions, the kinetic viscosity $\nu_{\text {kin }}$ and the collision viscosity $\nu_{\text {coll }}$. In two dimensions, approximate analytical expressions are $[34,35]$

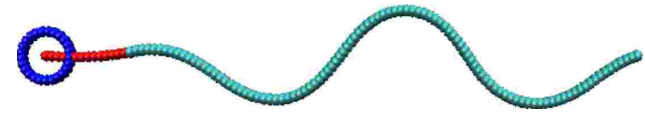

FIG. 1. (Color online) Two-dimensional model of sperm. The model consists of three parts, the head (blue), the midpiece (red), and the tail (cyan). Two sinusoidal waves are present on the beating tail.

$$
\begin{aligned}
& \frac{\nu_{\text {coll }}}{\sqrt{k_{B} T a^{2} / m}}=\frac{1}{12 h}(1-\cos \alpha)\left(1-\frac{1}{\rho}\right), \\
& \frac{\nu_{\text {kin }}}{\sqrt{k_{B} T a^{2} / m}}=h\left[\frac{1}{1-\cos \alpha} \frac{\rho}{\rho-1}-\frac{1}{2}\right],
\end{aligned}
$$

where $\rho$ is the average particle number in each box, $m$ is the mass of solvent particle, and $h=\Delta t \sqrt{k_{B} T / m a^{2}}$ is the rescaled mean free path. In this paper, we use $k_{B} T=1, m=1, a=1$, $\Delta t=0.025, \alpha=\pi / 2, \rho=10$. This implies, in particular, that the simulation time unit $\left(m a^{2} / k_{B} T\right)^{1 / 2}$ equals unity. With these parameters, the total kinematic viscosity of fluid is $\nu$ $=\nu_{\text {coll }}+\nu_{\text {kin }} \approx 3.02$. The size of the simulation box is $L_{x} \times L_{y}$, where $L_{x}=L_{y}=200 a$, four times the length of the sperm tail, if not indicated otherwise. Periodic boundary conditions are employed.

\section{B. Sperm model in two dimensions}

Although animal sperm differ from species to species, their basic structure is quite universal. Usually, a sperm consists of three parts: a head containing the genetic information, a beating long tail, and a midpiece to connect head and tail. Our two-dimensional sperm model, shown in Fig. 1, consists of these three parts. The head is constructed of $N_{\text {head }}=25$ particles, where neighboring particles are linked by springs of finite length $l_{0}=0.5 a$ with interaction potential

$$
V_{\text {bond }}(\mathbf{R})=\frac{1}{2} k\left(|\mathbf{R}|-l_{0}\right)^{2}
$$

where $\mathbf{R}$ is the bond vector, into a circle of radius $2 a$. Each of the head particles has a mass $m_{\text {head }}=20$. The midpiece consists of $N_{\text {mid }}=14$ particles of mass $m_{\text {mid }}=10$ connected by springs of length $l_{0}=0.5 \mathrm{a}$. The first particle of the midpiece, which is fixed to the center of the head, is connected with every particle on the head by a spring of length $l_{\text {head-mid }}$ $=2 a$, in order to maintain the circular shape of the head, as well as to stabilize the connection between head and midpart. The tail has $N_{\text {tail }}=100$ particles of mass $m_{\text {tail }}=10$, linked together by springs of length $l_{0}$. The spring constants are chosen to be $k_{\text {head-mid }}=10^{4}, k_{\text {head }}=10^{5}, k_{\text {mid }}=k_{\text {tail }}=2 \times 10^{5}$, where $k_{\text {head-mid }}$ is the spring constant for the connection of the head particles and the center, and $k_{\text {mid }}$ and $k_{\text {head }}$ are the spring constants for the tail and the mid-piece, respectively.

A bending elasticity is necessary for the midpiece and the tail to maintain a smooth shape in a fluctuating environment, and to implement the beating pattern. The bending energy is 


$$
\begin{aligned}
E_{\text {bend }} & =\sum_{i \in \text { mid }} \frac{1}{2} \kappa\left\{\mathbf{R}_{i+1}-\mathbf{R}_{i}\right\}^{2} \\
& +\sum_{i \in \text { tail }} \frac{1}{2} \kappa\left\{\mathbf{R}_{i+1}-\Re\left(l_{0} c_{s, \text { tail }}\right) \mathbf{R}_{i}\right\}^{2},
\end{aligned}
$$

where $\mathbf{R}_{i}$ is the bond vector connecting monomers $i$ and $(i$ $+1), \kappa$ denotes the bending rigidity, $\mathfrak{R}\left(l_{0} c_{s}\right)$ is a rotation matrix which rotates a vector anticlockwise by an angle $l_{0} c_{s}$, and $c_{s, \text { tail }}$ is the local spontaneous curvature of the tail of the $s$ th sperm. We choose $\kappa=10^{4}$, much larger than the thermal energy $k_{B} T=1$ to guarantee that the mechanical forces dominate the thermal forces. For the midpart, the spontaneous curvature vanishes, $c_{s, \text { mid }}=0 . c_{s, \text { tail }}$ is a variable changing with time $t$ and the position $x$ along the flagellum to create a propagating bending wave

$$
c_{s, \text { tail }}(x, t)=c_{0, \text { tail }}+A \sin \left[-2 \pi f_{s} t+q x+\varphi_{s}\right] .
$$

A detailed analysis of the beating pattern of bull sperm in Ref. [36] shows that a single sine mode represents the beat to a very good approximation. The wave number $q=4 \pi / l_{0} N_{\text {tail }}$ is chosen to mimic the tail shape of sea-urchin sperm [1], so that the phase difference between the first and the last particles of the tail is $4 \pi$, and two waves are present (see Fig. 1). $f_{s}$ is the beating frequency of the $s$ th sperm. The constant $c_{0, \text { tail }}$ determines the average spontaneous curvature of the tail. $\varphi_{s}$ is the initial phase of the first tail particle on the $s$ th sperm, and $A$ is a constant related to the beating amplitude. We choose $A=0.2$, which induces a beating amplitude $A_{\text {tail }}$ $=3.2 a$ of the tail. As $t$ increases, a wave propagates along the tail, pushing the fluid backward at the same time propelling the sperm forward. We keep $A, k, T_{s}$, and $\varphi_{s}$ constant for each sperm during a simulation. Although the spontaneous local curvature is prescribed, the tail is elastic and its configuration is affected by the viscous medium and the flow field generated by the motion of neighboring sperm.

In order to avoid intersections or overlaps of different sperm, we employ a shifted, truncated Leonard-Jones potential

$$
V(r)= \begin{cases}4 \epsilon\left[\left(\frac{\sigma}{r}\right)^{12}-\left(\frac{\sigma}{r}\right)^{6}\right]+\epsilon, & r<2^{1 / 6} \sigma, \\ 0, & r \geqslant 2^{1 / 6} \sigma\end{cases}
$$

between particles belonging to different sperm, where $r$ is the distance between two particles. Parameters $\sigma=1$ and $\epsilon$ $=13.75$ are chosen.

During the MPC streaming step, the equation of motion of the sperm particles is integrated by a velocity-Verlet algorithm, with a molecular-dynamics time step $\Delta t_{s}=5 \times 10^{-4}$, which is $1 / 50$ of the MPC time step $\Delta t$. The sperm only interacts with the fluid during the MPC collision step. This is done by sorting the sperm particles together with the fluid particles into the collision cells and rotating their velocities relative to the center-of-mass velocity of each cell.

Since energy is injected into the system by the actively beating tails, we employ a thermostat to keep the fluid temperature constant by rescaling all fluid-particle velocities in a collision box relative to its center-of-mass velocity after each collision step. This procedure has the advantage that the energy consumption per unit time of the sperm can be easily extracted through the rescaling of the particle velocities.

We start with a single-sperm system with $c_{0 \text {,tail }}=0$ and $f$ $=1 / 120$. With the other parameters given in the previous section, our sperm model swims smoothly forward with the velocity $u_{\text {single }}=0.016 \pm 0.001$. Because of its large size, the diffusion coefficient of a sperm due to the thermal fluctuations of the MPC fluid is very small, on the order of $10^{-4}$ [37]. This implies that the time the sperm needs to cover a distance of half the length of its flagellum by passive diffusion is more than a factor $10^{4}$ larger than the time to travel the same distance by active swimming. Therefore, diffusion plays a negligible role in our simulations. The energy consumption per unit time $P_{\text {single }}=25.2 \pm 2.4$ - corresponding to $P_{\text {single }} \simeq 3000 k_{B} T f$. Thus, we estimate a Reynolds number $\mathrm{Re}=2 A_{\text {tail }} u_{\text {single }} / \nu \simeq 0.03$ for our sperm model, where $A_{\text {tail }}$ $=3.2 a$ is the beating amplitude of the tail.

\section{TWO-SPERM SIMULATIONS}

\section{A. Symmetric sperm}

Two sperm, $S 1$ and $S 2$, are placed inside the fluid, initially with straight and parallel tails at a distance $d=5$ (i.e., with touching heads). They start to beat at $t=0$ with different phases $\varphi_{1}$ and $\varphi_{2}$. The initial positions of sperm do not matter too much, because two freely swimming sperm always have the chance to come close to each other after a sufficiently long simulation time. We consider two sperm with the same beat frequency $f=1 / 120$, and the same spontaneous curvature $c_{0 \text {, tail }}=0$.

In the dynamical behavior of these hydrodynamically interacting sperm, two effects can be distinguished, a short time "synchronization" and a longer time "attraction" process. If the initial phase difference $\Delta \varphi=\varphi_{2}-\varphi_{1}$ at time $t=0$ is not too large, an interesting effect denoted "synchronization" takes place, which is accomplished within a few beats. This process is illustrated in Figs. 2(a)-2(e) by snapshots at different simulation times. The synchronization time depends on the phase difference, and varies from about two beats for $\Delta \varphi=0.5 \pi$ (see Fig. 2) to about five beats for $\Delta \varphi=\pi$. A difference in swimming velocities adjusts the relative positions of the sperm. After a rapid transition, the velocities of two cells become identical once their flagella beat in phase. Because the initial distance between tails $d=5$ is smaller than the beating amplitude $2 A_{\text {tail }}=6.4 a$, the sperm tails can touch when they start to beat for $0.6 \pi<\Delta \varphi<1.4 \pi$. This geometrical effect is reduced by the hydrodynamic interaction, which affects the beating amplitude. In case contact occurs, it accelerates the synchronization. In order to avoid this direct interaction due to volume exclusion, we have also performed simulations of two sperm with initial distance $d=10$, and find the synchronized state achieved within several beats, as in the simulations with $d=5$. Thus, the synchronization effect is of purely hydrodynamic origin. Since the beating phase at time $t$ is determined by $f$ and $\varphi_{s}$, which are kept constant in our simulations, our model sperm can only achieve synchronization by adjusting the relative position. 


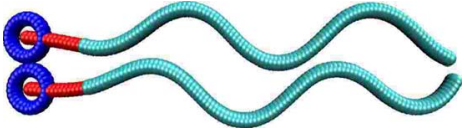

(a)

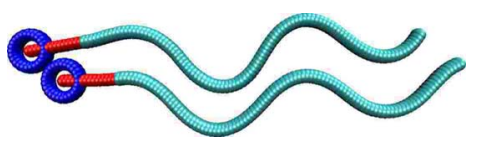

(d)

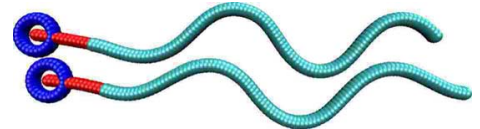

(b)

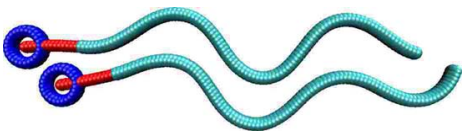

(e)

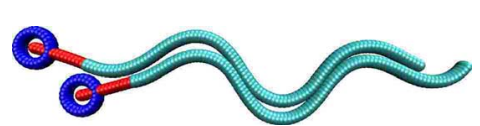

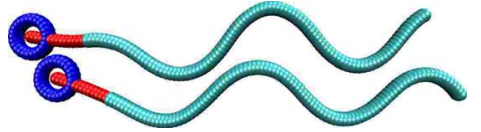

(c)

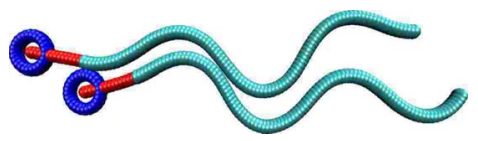

(f)

(g)

FIG. 2. (Color online) Snapshots of two sperm with phases $\varphi_{1}$ (upper), $\varphi_{2}$ (lower), and phase difference $\Delta \varphi=\varphi_{2}-\varphi_{1}=0.5 \pi$. (a) $t f$ $=1 / 6$ initial position; (b) $t f=2 / 3$; (c) $t f=7 / 6$; (d) $t f=5 / 3$; (e) $t f=13 / 6$; (f) $t f=10 \frac{1}{6}$; (g) $t f=100 \frac{1}{6}$. From (a) to (e), the synchronization process takes place. The tails are already beating in phase in (e). From (e) to (g), two synchronized sperm form a tight cluster due to hydrodynamic attraction.

Our results are in good agreement with the prediction of Taylor [5], based on an analytical analysis of twodimensional hydrodynamics, that the viscous stress between sinusoidally beating tails tends to force the two waves into phase. The same phenomenon has also been observed by Fauci and McDonald [6] in their simulations of sperm in the presence of boundaries, and has been called the "phaselocking" effect. A similar effect of undulating filaments immersed in a two-dimensional fluid at low Reynolds number was seen by Fauci in Ref. [38].

Synchronization is a fast process, which is achieved in at most ten beats in our simulations. Another hydrodynamic effect, which we denote "attraction," takes much longer time. Two synchronized and separated sperm gradually approach each other when they are swimming together, as if there was some effective attractive interaction between them. The only way in which the sperm can attract each other in our simulations is through the hydrodynamics of the solvent. This effect takes several ten beats to overcome the initial distance of $d=5$ between the tails. The final state of attraction, in which the sperm tails are touching tightly, is shown in Fig. 2(g).

Fauci and McDonald [6], did not see the hydrodynamic attraction, because they considered a sperm pair confined between two walls. As explained in Ref. [6], there is an evident tendency for a single sperm to approach the wall. When two parallel sperm are placed between the walls, there seems to be a critical initial distance between the sperm, below which synchronization occurs, and above which swimming towards the wall occurs. Our understanding is that, in their simulations, the viscous drag towards the walls was competing with the viscous attractive effect between sperm. Hence in some cases, they could only see a synchronization effect, and neither a clear towards-wall tendency nor a distinguishable attraction effect. The hydrodynamic attraction was masked by the presence of the walls.

To analyze the cooperating sperm pair in more detail, we choose the head-head distance $d_{h}$ to characterize the attraction and synchronization, because it is easy experimentally to track the head position. The dependence of $d_{h}$ on the phase difference is symmetric with respect to $\Delta \varphi=0$ because of the symmetry of the sperm structure. Thus, we show in Fig. 3 only results for $\Delta \varphi>0$. There is a plateau at about $d_{h}=5 a$ for $\Delta \varphi<0.4 \pi$, which corresponds to the sperm heads touching each other. For $\Delta \varphi>0.4 \pi, d_{h}$ increases linearly with $\Delta \varphi$. Finally, for $\Delta \varphi>1.5 \pi$, the phase difference is so large that the attraction is not strong enough to overcome the thermal fluctuations and pull the sperm close together. Although synchronization still occurs at the beginning, the two sperm leave each other soon after.

Riedel et al. [16] also see such a linear relation in their experiments of sea-urchin sperm vortices. They define the beating phase of a sperm by its head oscillation, and an angular position of the sperm head within the vortex. In this way, the beating phase difference of the sperm in the same

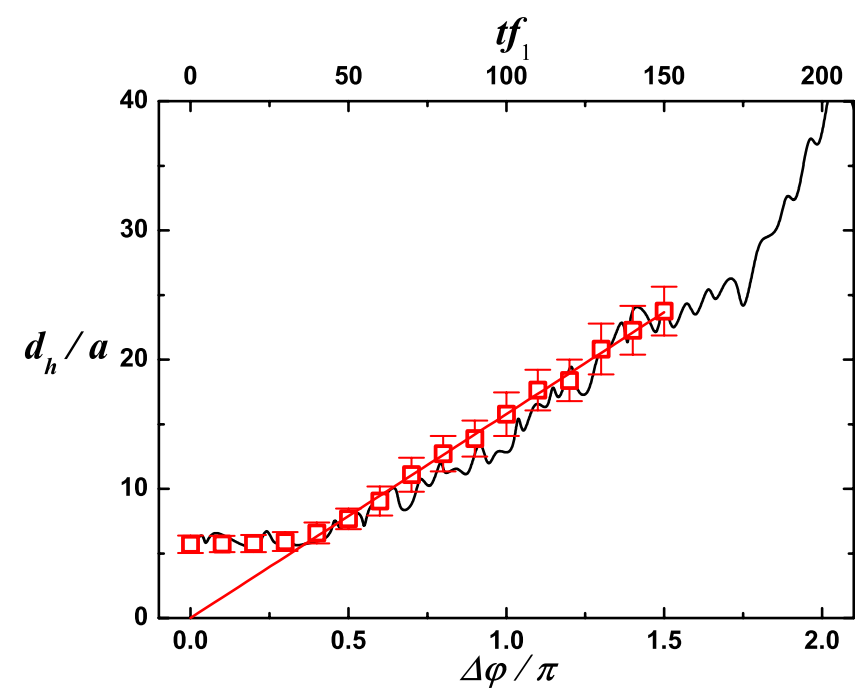

FIG. 3. (Color online) Head-head distance $d_{h}$ of two cooperating sperm. Simulation data are shown for fixed phase difference (red, $\square$ ), with error bars denoting the standard deviation. The interpolating (red) line is a linear fit for $0.4 \pi<\Delta \varphi<1.5 \pi$. The distance $d_{h}$ is also shown as a function of time $t$ (top axis) in a simulation with a $0.5 \%$ difference in the beat frequencies of the two sperm (solid line). 


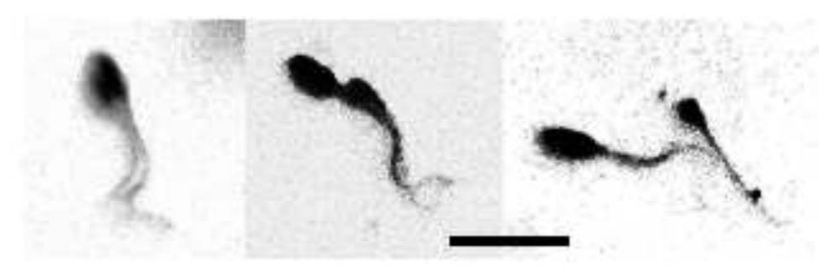

FIG. 4. Snapshots of two synchronized human sperm in experiment at different times $[39,40]$. (Left) Two sperm with initially well synchronized tails and very small phase difference. (Middle) The sperm are still swimming together and are well synchronized after $4 \mathrm{~s}$; a phase difference has developed. (Right) The sperm begin to depart after $7 \mathrm{~s}$. The scale bar corresponds to a length of $25 \mu \mathrm{m}$.

vortex was found to have a linear relation with the angular position difference, which corresponds to the head-head distance in our simulations.

So far, we have considered sperm with a single beat frequency. In nature, sperm of the same species always have a wide distribution of beat frequencies. For example, the beat frequency of sea-urchin sperm ranges from $30-80 \mathrm{~Hz}$ [1], and the frequency of bull sperm ranges from $20-30 \mathrm{~Hz}$ [36]. Thus, we assign different beat frequencies to two sperm, $f_{1}$ $=1 / 120$ and $f_{2}=1 / 119.4$, corresponding to $\Delta f / f_{1} \approx 0.5 \%$, but set the same initial phases $\varphi_{s}=0$. This implies that the phase difference of the beats between the two sperm increases linearly in time,

$$
\Delta \varphi=2 \pi\left(f_{2}-f_{1}\right) t .
$$

Figure 3 shows the head-head distance versus time. It agrees very well with the data for fixed phase differences. At $t f_{1}$ $=150$ where $\Delta \varphi \simeq 1.5 \pi$, the sperm trajectories begin to depart.

Figure 4 shows two cooperating human sperm swimming in an in vitro experiment near a glass substrate $[39,40]$. The two sperm swim together for more than $6 \mathrm{~s}$ at a beat frequency of approximately $8 \mathrm{~Hz}$. Their tails remain synchronized during this time, while the head-head distance and phase difference increases with time (see Fig. 4). After a while, the sperm leave each other because the phase difference becomes too large. There is no indication of a direct adhesive interaction between the sperm.

An interesting question is whether the cooperation of a sperm pair reduces the energy consumption. Figure 5 displays the energy consumption of two sperm with the same beat frequency $f=1 / 120$ as a function of the phase difference. The energy consumption $P$ is nearly constant at small phase difference. It increases for $\Delta \varphi \geqslant 0.5 \pi$ roughly linearly until it reaches another plateau for $\Delta \varphi \geqslant 1.5 \pi$. The second plateau corresponds to two sperm swim separately, so that energy consumption is twice the value of a single sperm. Our results are in agreement with the conclusion of Taylor [5] that less energy is dissipated in the fluid if the tails are synchronized.

Figure 5 also shows the energy consumption of two sperm with $f_{1}=1 / 120, f_{2}=1 / 119.4$, and $\varphi_{1}=\varphi_{2}=0$ as a function of time $t$. In this simulation, we start with two sperm which are parallel and at a distance $d=5$. For $t f_{1}<25$, the energy con-

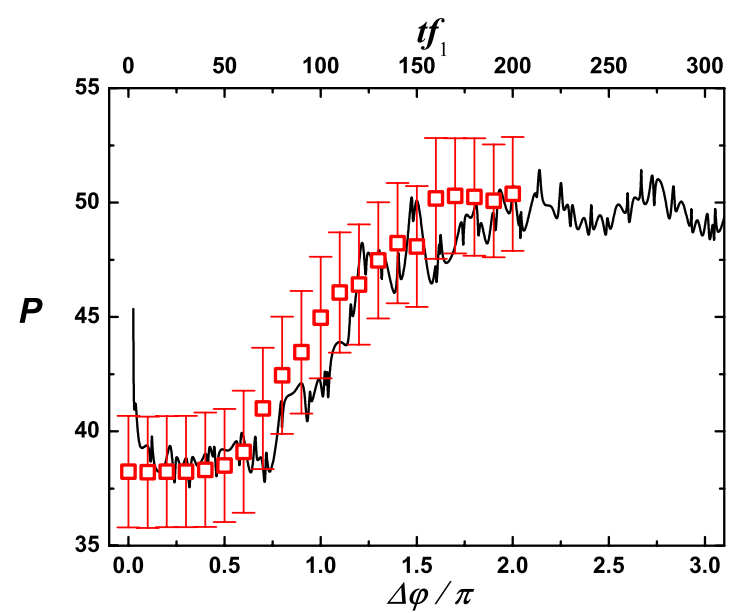

FIG. 5. (Color online) Energy consumption per unit time $P$ of two cooperating sperm. Symbols show simulation data for fixed phase difference (red, $\square$ ), where error bars denote the standard deviation. $P$ versus time $t$ in a simulation with a $0.5 \%$ difference in the frequencies of two sperm (solid black line).

sumption decreases as the sperm are approaching each other. The data agrees quantitatively very well with results for constant $\Delta \varphi$, and reaches a plateau when the cooperating sperm pair departs.

The synchronization and attraction also exists in our simulation of swimming flagella without heads. In this case, the time-reversal symmetry of Stokes flow implies that no synchronization nor attraction is possible at zero Reynolds number. In our simulations, the thermal fluctuations and a finite Reynolds number break the time-reversal symmetry.

\section{B. Asymmetric sperm}

In nature, sperm have an abundance of different shapes. In particular, these shapes are typically not perfectly symmetric. The asymmetric shape can cause a curvature of the sperm trajectory $[7,41]$. For example, sea-urchin sperm uses the spontaneous curvature of the tail to actively regulate the sperm trajectory for chemotaxis $[42,43]$. In our simulations, we impose an asymmetry of the tail by employing a nonzero spontaneous curvature $c_{0 \text {,tail }}$.

We consider curved sperm tails, with $c_{0, \text { tail }}=0.04 / a$, which results in a mean curvature of the trajectory of a single sperm of $c_{t} a=0.041 \pm 0.009$. For sperm with curved tails, the head-head distance $d_{h}(\Delta \varphi)$ is not symmetric about $\Delta \varphi=0$, as shown in Fig. 6. Here $\Delta \varphi$ is defined as the phase of the sperm on the inner circle minus the phase of the sperm on the outer circle. The steric repulsion of the heads causes a plateau of the head-head distance at $d_{h}=5$ for small phase differences $\Delta \varphi$, as in Fig. 3 for symmetric sperm. For $\Delta \varphi$ $<-\pi / 2$ and $\Delta \varphi>\pi / 4$, the head distance increases linearly with increasing phase difference, with a substantial difference of the slopes for $\Delta \varphi<0$ and $\Delta \varphi>0$, see Fig. 6. The two sperm depart when $\Delta \varphi>0.7 \pi$. For $\Delta \varphi \lesssim-2.0 \pi$, the sperm pair briefly loses synchronicity, but then rejoins with a new phase difference $\Delta \varphi^{\prime}=\Delta \varphi+2 \pi$.

The energy consumption $P$ for sperm with spontaneous curvature (see Fig. 7) also increases sharply at $\Delta \varphi=0.8 \pi$ and 


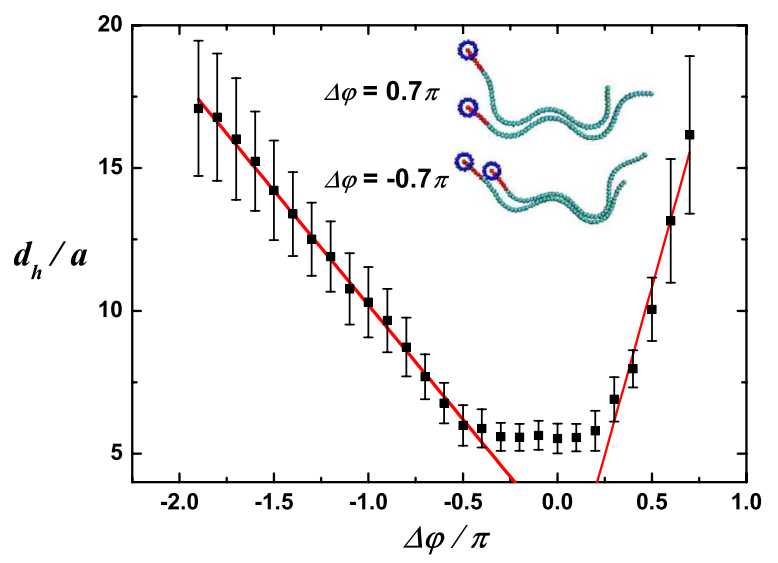

FIG. 6. (Color online) Head-head distance $d_{h}$ of two cooperating sperm with spontaneous curvature $c_{0 \text {,tail }}=0.04 / a$ as a function of the phase difference $\Delta \varphi$. The error bars represent standard deviations. Lines are linear fits to the data in the range $-1.9 \pi<\Delta \varphi<-0.5 \pi$ and $0.3 \pi<\Delta \varphi<0.7 \pi$, respectively. The inset shows two typical conformations with positive and negative phase difference.

stays at the plateau with $P=51.0 \pm 2.8$ for larger $\Delta \varphi$, as the sperm are swimming separately. However, for $\Delta \varphi<-\pi / 2, P$ increases rather smoothly until the cooperation is lost for large phase differences. We conclude that the strong curvature of the tail breaks the symmetry of the head-head distance $d_{h}$ and the energy consumption $P$ in $\Delta \varphi$, but the effect of synchronization and attraction is still present and play an important role in the cooperation of sperm pairs.

\section{MULTISPERM SYSTEMS}

When two sperm with the same beating period happen to get close and parallel, they interact strongly through hydrodynamics and swim together. With this knowledge of hydrodynamic interaction between two sperm, we now study a system of 50 sperm in a simulation box of $200 \times 200$ collision boxes, which corresponds to a density of about three sperm per squared sperm length. The initial position and orientation for each sperm are chosen randomly. Considering

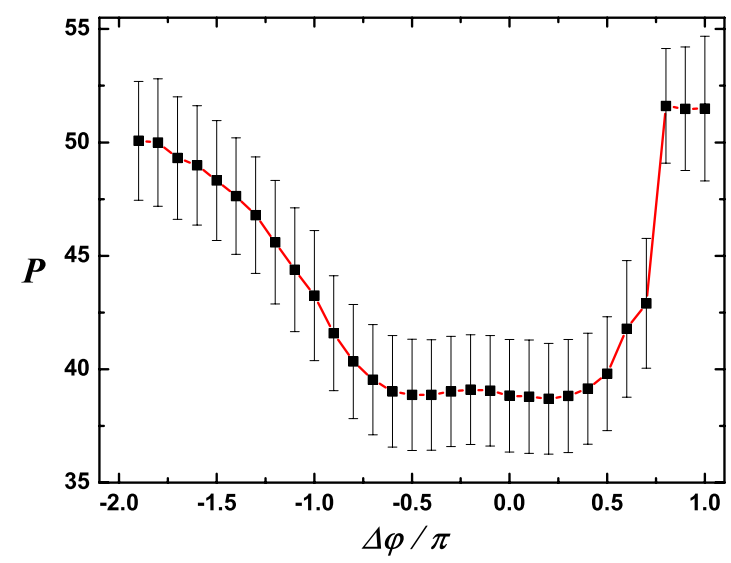

FIG. 7. (Color online) Energy consumption per unit time $P$ versus phase difference $\Delta \varphi$ of two sperm with spontaneous curvature $c_{0, \text { tail }}=0.04 / a$ of their tails. The error bars represent standard deviations.

that in real biological systems the beat frequency is not necessary the same for all sperm, we perform simulations with Gaussian-distributed beating frequencies. The initial phases of all sperm are $\varphi_{s}=0$.

We consider a system of symmetric sperm. Figure 8 shows some snapshots of systems with different width $\delta_{f}$ $=\left\langle(\Delta f)^{2}\right\rangle^{1 / 2} /\langle f\rangle$ of the Gaussian frequency distribution. Here, $\left\langle(\Delta f)^{2}\right\rangle$ is the mean square deviation of the frequency distribution and $\langle f\rangle=1 / 120$ is the average frequency.

For $\delta_{f}=0$, once a cluster has formed, it does not disintegrate without a strong external force. A possible way of break-up is by bumping head-on into another cluster. For $\delta_{f}>0$, however, sperm cells can leave a cluster after sufficiently long time, since the phase difference to other cells in the cluster increases in time due to the different beat frequencies (compare Sec. III A). At the same time, the cluster size can grow by collecting nearby free sperm or by merging with other clusters. Thus, there is a balance between cluster formation and break-up, as shown in the accompanying movie [44]. Obviously, the average cluster size is smaller for large $\delta_{f}$ than for small $\delta_{f}$ (see Fig. 8).

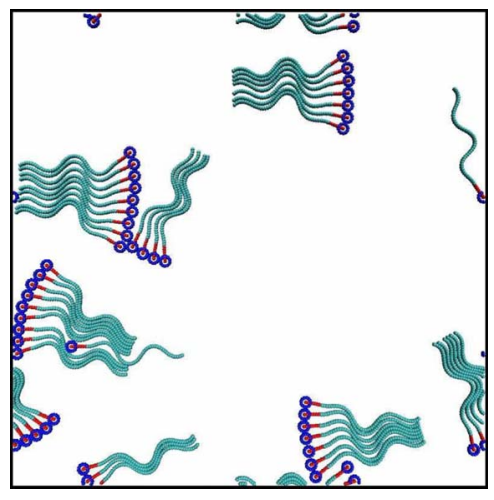

(a)

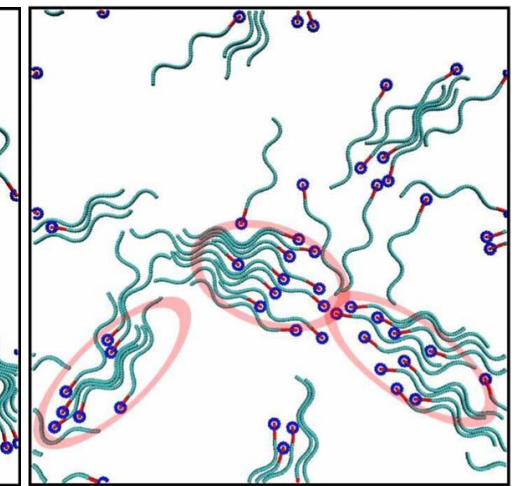

(b)

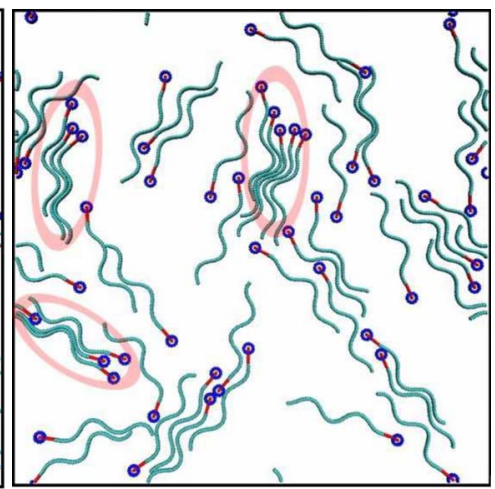

(c)

FIG. 8. (Color online) Snapshots from simulations of 50 symmetric sperm with different widths $\delta_{f}$ of a Gaussian distribution of beating frequencies. (a) $\delta_{f}=0$; (b) $\delta_{f}=0.9 \%$; (c) $\delta_{f}=4.5 \%$. The red ellipses in (b) and (c) indicate large sperm clusters. The black frames show the simulation boxes. Note that we employ periodic boundary conditions. 


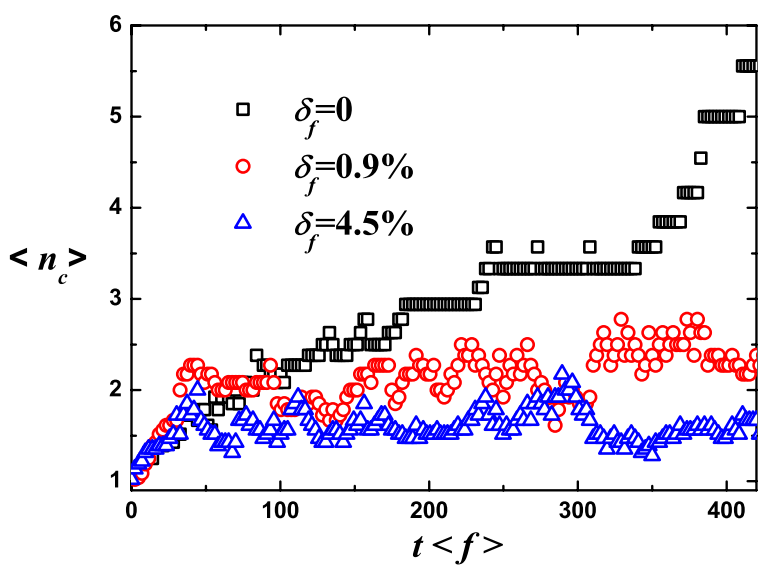

FIG. 9. (Color online) Time dependence of the average cluster size $\left\langle n_{c}\right\rangle$ in a system of 50 symmetric sperm with various widths $\delta_{f}$ of the frequency distribution, as indicated.

To analyze the multisperm systems, we define a cluster as follows. If the angle between vectors from the last to the first bead of the tails of two sperm is smaller than $\pi / 6$, and at the same time the nearest distance between the tails is smaller than $4 a$, which is approximately $1 / 10$ of the length of the tail, then we consider these two sperm to be in the same cluster. By this definition, we find the evolution of the average cluster size $\left\langle n_{c}\right\rangle$ shown in Fig. 9. Here, $\left\langle n_{c}\right\rangle$ is the average number of sperm in a cluster

$$
\left\langle n_{c}\right\rangle=\sum_{n_{c}} n_{c} \Pi\left(n_{c}\right),
$$

where $\Pi\left(n_{c}\right)$ is the (normalized) cluster-size distribution. For $\delta_{f}=0$, the average cluster size continues to increase with time. Both systems in Fig. 9 with $\delta_{f}>0$ reach a stationary cluster size after about 50 beats. The stationary cluster size is plotted in Fig. 10 as a function of the width $\delta_{f}$ of the frequency distribution. We find a decay with a power law

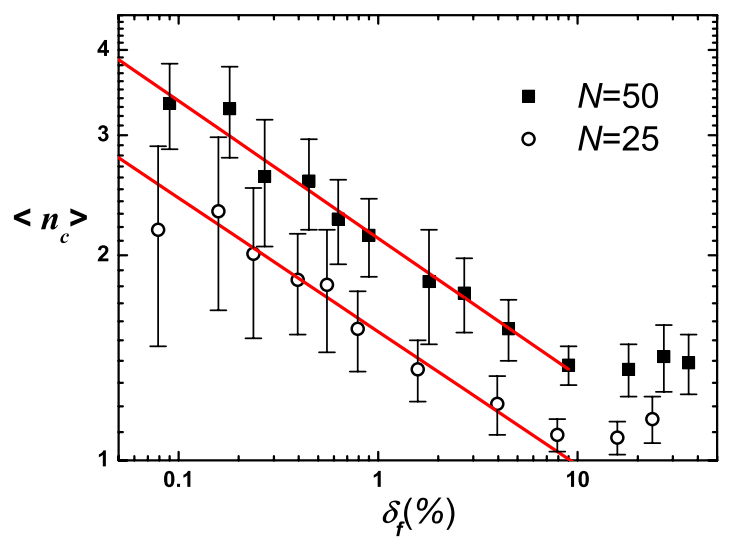

FIG. 10. (Color online) Dependence of the average stationary cluster size $\left\langle n_{c}\right\rangle$ on the width of the frequency distribution $\delta_{f}$. Data are shown for a 50 -sperm system $(\boldsymbol{\square})$ and a 25 -sperm system $(\bigcirc)$. The lines indicate the power-law decays $\left\langle n_{c}\right\rangle=2.12 \delta_{f}^{-0.201}$ (upper) and $\left\langle n_{c}\right\rangle=1.55 \delta_{f}^{-0.196}$ (lower).

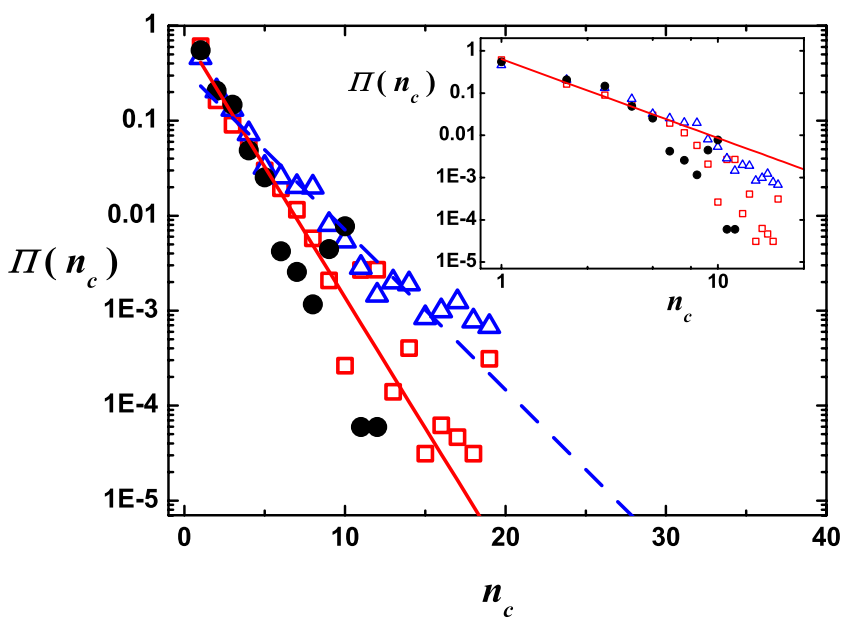

FIG. 11. (Color online) Cluster size distribution $\Pi\left(n_{c}\right)$. Data are shown for 25 sperm in a $200 \times 200 a^{2}$ box $(-100$ sperm in a $400 \times 400 a^{2}$ box $(\square)$ [note that both systems have the same sperm density], and 50 sperm in a $200 \times 200 a^{2}$ box $(\triangle)$. The lines correspond to an exponential distribution. The inset shows the same data in a double-logarithmic representation. The line indicates a power law $n_{c}^{-1.8}$.

$$
\left\langle n_{c}\right\rangle \sim \delta_{f}^{-\gamma}
$$

with $\gamma=0.20 \pm 0.01$. The error for $\gamma$ is estimated from a fit of the data for both 50 -sperm and 25 -sperm systems. The negative power law indicates that the cluster size diverges when $\delta_{f} \rightarrow 0$. This tendency is also implied by the continuously increasing cluster size for $\delta_{f}=0$ in Fig. 9. The cluster-size distribution in the stationary state is shown in Fig. 11. Cluster-size distributions have been studied in much simpler systems of self-propelled particles, such as point particles with a constant magnitude of their velocities, which adjust their traveling direction to the direction of their neighbors [45]. In such simplified models, a power-law decay of the cluster-size distribution with an exponent in the range of 1.5 to 1.9 have been found [46], followed by a rapid decay for large cluster sizes due to finite-size effects. Similarly, in a system of self-propelled rods with volume exclusion, a crossover from power-law behavior at small cluster sizes to a more rapid decay for large cluster sizes has also been found [47]. A power-law decay of the cluster-size distribution is indeed consistent with our results for smaller cluster sizes, as shown in the inset of Fig. 11. The rather similar value of the exponent with that of Ref. [46] is probably fortuitous. We attribute the exponential decay of the cluster-size distribution for larger cluster size, which is apparent in Fig. 11, to finitesize effects. Simulations of larger system sizes are required to confirm this conclusion.

To analyze the energy consumption of sperm clusters, we consider a special case where sperm of the same frequency are prearranged to pack tightly and to be synchronized, as shown in Fig. 12(a). A simple linear relationship between the energy consumption of the sperm cluster and the cluster size is shown in Fig. 12(a). From the linear fit of the data, we obtain an energy consumption per sperm for an infinitely large cluster $P / n_{c}=13.7$. Thus, a freely swimming sperm can 

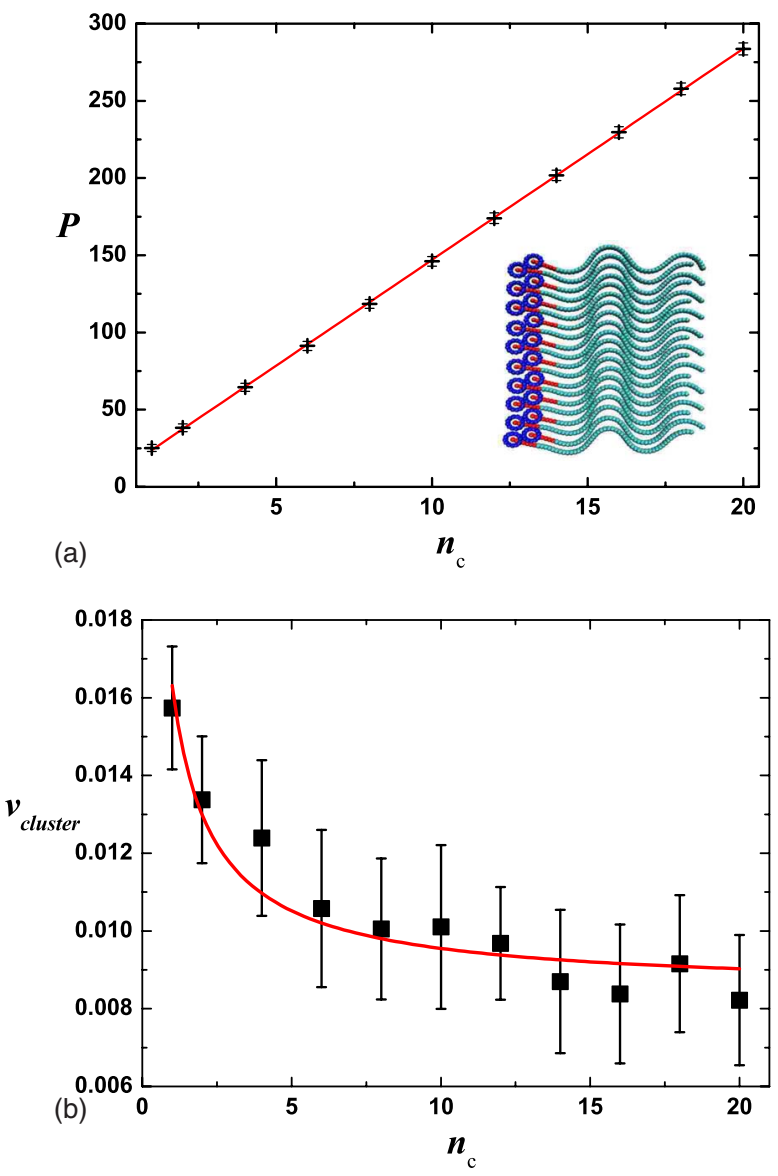

FIG. 12. (Color online) (a) Energy consumption per unit time $P$ of sperm clusters as a function of cluster size $n_{c}$. Symbols indicate simulations results. The fit line (red) is given by $P=13.7 n_{c}+10.1$. The inset shows an illustration of an arranged cluster of 20 sperm. (b) Center-of-mass speed of sperm clusters as a function of cluster size $n_{c}$. The fit line (red) is given by $v=0.00334 \sqrt{6.42+16.4 / n_{c}}$.

reduce its energy consumption by almost a factor 2 by joining a cluster.

The swimming speed of a sperm cluster decreases slowly with increasing sperm number, as shown in Fig. 12(b). When flagella are very close, with distances smaller than the size of a MPC collision box, hydrodynamic interactions are no longer properly resolved. Instead, the collision procedure yields a sliding friction for the relative motion of neighboring flagella. Thus, the energy of the beat is not only used for propulsion, but also to overcome the sliding friction. The energy consumption of tail-tail friction is proportional to the number of neighbor pairs, and the hydrodynamic resistance of moving the whole cluster is proportional to the cluster size and speed. Thus, the total energy consumption can be written as

$$
P=C n_{c} v^{2}+p_{f}\left(n_{c}-1\right),
$$

where $p_{f}$ is the energy consumption due to tail-tail friction, and $C$ is a constant. With the relation $P=13.7 n_{c}+10.1$ obtained above, the data for the cluster speed can be fitted to Eq. (14), which yields $p_{f}=7.28$ and $C=8.96 \times 10^{4}$. Thus, the cluster speed reaches a nonzero asymptotic value $[(13.7$ $\left.\left.-p_{f}\right) / C\right]^{1 / 2} \simeq 0.0085$. for large cluster size, about a factor 2 slower than a single sperm.

\section{SUMMARY AND DISCUSSION}

We have simulated the hydrodynamic interaction between sperm in two dimensions by the MPC method. Two effects of the hydrodynamic interaction were found in our simulations. First, when two sperm are close in space and swimming parallel, they synchronize their tail beats by adjusting their relative position. This process can be accomplished in a very short time, less than 10 beats. Second, two synchronized sperm have a tendency to get close and form a tight pair. This process takes much longer time then synchronization. It usually takes about 100 beats to overcome a distance of $1 / 10$ tail length between sperm in our simulations.

These hydrodynamic effects favor the cooperation of sperm in motile clusters. For a multisperm system, the average cluster size diverges if all sperm have the same beating frequency. A distribution of frequencies leads to a stationary cluster-size distribution with a finite average cluster size, which decreases with a power law of the variance of the frequency distribution. Furthermore, the average cluster size increases with increasing sperm density. The probability to find a cluster decreases with a power law for small cluster sizes; an exponential decay for large cluster sizes is attributed to finite-size effects.

In sperm experiments, large bundles have been found in some species, such as fish flies $[11,13]$ and wood mouse $[12,15]$. For fish-fly sperm, this has been attributed to some agglutination of the sperm heads to keep the size and structure of the bundles. Wood mouse sperm were released into an in vitro laboratory medium, initially in single cell suspension [12]. Within $10 \mathrm{~min}$, large bundles containing hundreds or thousands of sperm were formed as motile "trains" of sperm. Motile bundles of 50-200 sperm were also found in the aftermating female's body, as well as many non-motile single sperm. The hook structure on the head of wood mouse sperm is believed to favor the formation of such huge cluster in in vitro experiments.

In our simulations, sperm clusters are always seen, e.g., as marked in Fig. 8, after the system has reached a dynamically balanced state of cluster sizes. Thus, we predict that hydrodynamic synchronization and attraction play an important role in the cluster formation of healthy and motile sperm, such as the bundles and trains observed for fish-fly and wood-mouse sperm at high concentrations, respectively. Furthermore, since the cluster size decreases with increasing width $\delta_{f}$ of the distribution of beat frequencies, our results are consistent with the experimental observation that if the sperm are hyperactivated [12], which is an abnormal beat mode, or if some sperm are dead, the clusters fall apart.

\section{ACKNOWLEDGMENTS}

We thank U. Benjamin Kaupp, Luis Alvarez (CAESAR Bonn), and Luru Dai (INB-1, Research Center Jülich) for 
stimulating discussions and sharing of their experimental data. Y.Y. acknowledges financial support by the Interna- tional Helmholtz Research School on Biophysics and Soft Matter ("BioSoft").
[1] J. Gray and G. J. Hancock, J. Exp. Biol. 32, 802 (1955).

[2] L. J. Fauci and R. Dillon, Annu. Rev. Fluid Mech. 38, 371 (2006).

[3] S. Camalet, F. Jülicher, and J. Prost, Phys. Rev. Lett. 82, 1590 (1999).

[4] C. J. Brokaw, Math. Methods Appl. Sci. 24, 1351 (2001).

[5] G. I. Taylor, Proc. R. Soc. London, Ser. A 209, 447 (1951).

[6] L. J. Fauci and A. McDonald, Bull. Math. Biol. 57, 679 (1995).

[7] J. Elgeti, Ph.D. thesis, Universität zu Köln, Köln, 2007.

[8] L. Rothschild, Nature (London) 198, 1221 (1963).

[9] D. M. Woolley, Reproduction (Bristol, U.K.) 126, 259 (2003).

[10] A. P. Berke, L. Turner, H. C. Berg, and E. Lauga, Phys. Rev. Lett. 101, 038102 (2008).

[11] F. Hayashi, J. Insect Physiol. 42, 859 (1996).

[12] H. D. M. Moore and D. A. Taggart, Biol. Reprod. 52, 947 (1995).

[13] F. Hayashi, Funct. Ecol. 12, 347 (1998).

[14] S. Immler, H. D. M. Moore, W. G. Breed, and T. R. Birkhead, PLoS ONE 2, e170 (2007).

[15] H. D. M. Moore, K. Dvoráková, N. Jenkins, and W. G. Breed, Nature (London) 418, 174 (2002).

[16] I. H. Riedel, K. Kruse, and J. Howard, Science 309, 300 (2005).

[17] E. M. Purcell, Am. J. Phys. 45, 3 (1977).

[18] T. Ishikawa and M. Hota, J. Exp. Biol. 209, 4452 (2006).

[19] S. Nasseri and N. Phan-Thien, Comput. Mech. 20, 551 (1997).

[20] M. Kim, J. Bird, A. J. van Parys, K. S. Breuer, and T. R. Powers, Proc. Natl. Acad. Sci. U.S.A. 100, 15481 (2003).

[21] M. J. Kim and T. R. Powers, Phys. Rev. E 69, 061910 (2004).

[22] M. Reichert and H. Stark, Eur. Phys. J. E 17, 493 (2005).

[23] R. Dreyfus et al., Nature (London) 437, 862 (2005).

[24] E. Gauger and H. Stark, Phys. Rev. E 74, 021907 (2006).

[25] E. E. Keaveny and M. R. Maxey, Phys. Rev. E 77, 041910 (2008).

[26] A. Najafi and R. Golestanian, Phys. Rev. E 69, 062901 (2004).

[27] C. M. Pooley, G. P. Alexander, and J. M. Yeomans, Phys. Rev. Lett. 99, 228103 (2007).

[28] A. Malevanets and R. Kapral, J. Chem. Phys. 110, 8605 (1999).

[29] A. Malevanets and R. Kapral, J. Chem. Phys. 112, 7260
(2000).

[30] A. Lamura, G. Gompper, T. Ihle, and D. M. Kroll, Europhys. Lett. 56, 319 (2001).

[31] M. Ripoll, K. Mussawisade, R. G. Winkler, and G. Gompper, Europhys. Lett. 68, 106 (2004).

[32] T. Ihle and D. M. Kroll, Phys. Rev. E 63, 020201(R) (2001).

[33] T. Ihle and D. M. Kroll, Phys. Rev. E 67, 066705 (2003).

[34] N. Kikuchi, C. M. Pooley, J. F. Ryder, and J. M. Yeomans, J. Chem. Phys. 119, 6388 (2003).

[35] E. Tüzel, M. Strauss, T. Ihle, and D. M. Kroll, Phys. Rev. E 68, 036701 (2003).

[36] I. H. Riedel-Kruse, A. Hilfinger, J. Howard, and Frank Jülicher, HFSP J. 1, 192 (2007).

[37] K. Mussawisade, M. Ripoll, R. G. Winkler, and G. Gompper, J. Chem. Phys. 123, 144905 (2005).

[38] L. J. Fauci, J. Comput. Phys. 86, 294 (1990).

[39] Luis Alvarez, Luru Dai, and U. Benjamin Kaupp (private communication).

[40] The temperature in the human sperm experiment was about $35-37{ }^{\circ} \mathrm{C}$. The pictures were taken by fluorescence microscopy. The sperm was loaded with Fluo4 (concentration 10 millimole/liter), a calcium indicator. The measurement stage was moved to keep the swimming sperm in the field of view.

[41] J. Elgeti and G. Gompper, NIC proceedings, Vol. 39 of NIC Series, edited by G. Münster, D. Wolf, and M. Kremer (Neumann Institute for Computing, Jülich, 2008), pp. 53-61.

[42] T. Strunker, I. Weyand, W. Bonigk, Q. Van, A. Loogen, J. E. Brown, N. Kashikar, V. Hagen, E. Krause, and U. B. Kaupp, Nat. Cell Biol. 8, 1149 (2006).

[43] B. M. Friedrich and F. Jülicher, Proc. Natl. Acad. Sci. U.S.A. 104, 13256 (2007).

[44] See EPAPS Document No. E-PLEEE8-78-081811 for movies of the dynamics of cluster formation and breakup in the stationary state. For more information on EPAPS, see http:// www.aip.org/pubservs/epaps.html

[45] T. Vicsek, A. Czirok, E. Ben-Jacob, I. Cohen, and O. Shochet, Phys. Rev. Lett. 75, 1226 (1995).

[46] C. Huepe and M. Aldana, Phys. Rev. Lett. 92, 168701 (2004).

[47] F. Peruani, A. Deutsch, and M. Bär, Phys. Rev. E 74, 030904(R) (2006). 